\title{
The Effect of Midazolam Together with Acetaminophen in Premedication: A Study in a Tertiary Care Hospital, Pabna, Bangladesh
}

\author{
Dr. Ataur Rahman ${ }^{1 *}$, Dr. Mohammad Faisal Zinnat ${ }^{2}$, Dr. Md. Sirajul Islam³ ${ }^{3}$ Dr. Mohammad Mostafizur Rahman \\ ${ }^{1}$ Senior Consultant (Anaesthesiology), 250-Bedded General Hospital, Pabna, Bangladesh \\ ${ }^{2}$ Anesthesiologist, 250-Bedded General Hospital, Pabna, Bangladesh \\ ${ }^{3}$ Assistant professor, OSD, DGHS, Mohakhali, Dhaka-1212 attachment of Pabna Medical College \& Hospital, Pabna, Bangladesh \\ ${ }^{4}$ Associate Professor \& Head, Dept. of Anesthesiology \& ICU, Ashiyan Medical College Hospital, Barua Khilkhet, Dhaka, Bangladesh
}

DOI: $10.36347 /$ sjams.2020.v08i06.045

| Received: 19.06.2020 | Accepted: 26.06.2020 | Published: 30.06 .2020

*Corresponding author: Dr. Ataur Rahman

Abstract

Background: Premedication is the administration of medication before anesthesia. It is used to prepare the patient for anesthesia and to help provide optimal conditions for surgery. In premedication, Sedative premedication is widely administered before surgery. Midazolam has been used extensively worldwide for pharmacological premedication. The aim of this study was to determine the effect of Midazolam together with Acetaminophen in premedication. Methods \& Materials: This was a descriptive observational study was conducted at the department of Anaesthesiology in 250 bedded general hospital, Pabna, Bangladesh during the period from the study during January 2017 to December, 2018. A total of 100 patients, below 8 years of age and either sex who were scheduled for elective surgery or diagnostic procedures requiring anaesthesia techniques and prescribed anxiolytic premedication with Midazolam by the anaesthesiologist were included in the study during January, 2017 to December, 2018. The patients were assessed by the attending anaesthetist, and given the modified Yale scale. Patients were taken on a stretcher to the operating room, and after setting up basic monitoring, inhaled induction was initiated using sevoflurane. At that point (1min), the Richmond-RASS sedation-agitation scale was applied to determine the sedation of the patients. Results: A total of 100 children included in this study. The mean BMI of the children were $15 \pm 3$. The age of the children was $>08$ years. Of them, $30 \%$ were females and $70 \%$ were males. The application of the Yale scale showed adequate sedation and anxiolytics in $60 \%$ of the patients. When the sedation-anxiolytics scale was applied at the time the children were separated from their parents, it showed that $61 \%$ of the children were indifferent or quiet, anious, not violent were $15 \%$, frequent movement were $08 \%$, agglessive, violent were $05 \%$, very agitated were $04 \%$, sleepy were $03 \%$, deep sedation was $01 \%$ At this stage, there was no evaluation of the time elapsed from the moment the Midazolamacetaminophen mix was given. The Richmond-RASS scale applied at the time of induction showed that $65 \%$ of the patients had adequate sedation (score between -1 and 0 ), which resulted in easy and quiet acceptance of the facemask. Conclusion: From this study it is vivid that premedication with compounded Midazolam plus Acetaminophen used is useful. It reduces anxiety at the time when the children are separated from their parents, and it leads to good acceptance of inhaled anaesthesia induction, improving the whole experience, both for the children as well as for the parents.

Keywords: Premedication, Anesthesia, Midazolam, Acetaminophen.

Copyright @ 2020: This is an open-access article distributed under the terms of the Creative Commons Attribution license which permits unrestricted use, distribution, and reproduction in any medium for non-commercial use (NonCommercial, or CC-BY-NC) provided the original author and source are credited.

\section{INTRODUCTION}

Midazolam is a water soluble benzodiazepine, a member of a new class of imidazobenzodiazepine derivatives $[1,2]$. It is water soluble at $\mathrm{pH}<4$ but becomes highly lipid soluble at physiologic $\mathrm{pH}$. Water solubility minimizes pain at the injection site. The lipid solubility ensures rapid distribution in tissues. The benzodiazepines exhibit anxiolytic, sedative, amnesic and anticonvulsant properties. They differ mainly in their pharmacokinetic properties, some being relatively short acting whereas others are only slowly eliminated. Midazolam is more potent, has a faster onset and shorter duration of action slowly eliminated Midazolam is more potent, has a faster onset and shorter duration of action Premedication is using medication before some other therapy (usually surgery or chemotherapy) to prepare for that forthcoming therapy[3]. Typical examples include premeditating with a sedative or 
analgesic before surgery; using prophylactic (preventive) antibiotics before surgery; and using antiemetic or antihistamines before chemotherapy [4]. Now-a-days premedication with sedative drugs is often used in paediatric practice as one of the modalities to reduce preoperative anxiety in children undergoing surgery. Sedative premedication is a successive practice in paediatric anaesthesia administrations [5, 6]. It has been appeared to lessen tension in the children and their folks at the time they are taken to the working room, the pain relieving necessities, and the time required for breathed in acceptance and ortracheal intubation. The utilization of pharmacological premedication has appeared to be more practical for controlling uneasiness when contrasted and the utilization of nonpharmacological means, for example, the nearness of the guardians amid acceptance, or behavioral readiness programs before surgery. Midazolam has been utilized widely for pharmacological premedication, with sufficient results [7, 8]. At the Red Cross Kids' College Clinic in Manizales, an aggravated planning has been utilized since 2000, comprising of a blend of Midazolam ampoules $15 \mathrm{mg} / 3 \mathrm{ml}$ or more acetaminophen suspension $150 \mathrm{mg} / 5 \mathrm{ml}$. At the establishment, the blend is known as "midazophen", and after it is readied it comprises of Midazolam $1 \mathrm{mg} / \mathrm{ml}$ and acetaminophen $24 \mathrm{mg} / \mathrm{ml}$. The measurement utilized is a large portion of the body weight in cubic centimeters, which is equivalent to $0.5 \mathrm{mg} / \mathrm{kg}$ of oral Midazolam and $12 \mathrm{mg} / \mathrm{kg}$ of acetaminophen. The dosage is offered orally to patients booked for elective surgery [9]. Premedication is the administration of medication before anaesthesia. It is used to prepare the patient for anaesthesia and to help provide optimal conditions for surgery. In premedication, Sedative premedication is widely administered before surgery. The common drugs are being used for premedication, Ketamine, doses$\mathrm{mg} / \mathrm{kg}$, PO-2-10 IV. 25-1.0 - IM 1-4.0, Chloral Hydrate, PO: 25-100, Promethazine, PO 0.1-1.0, Diazepam, PO 0.1-0.4. So, the researcher, purposively, wanted to determine the effect of Midazolam together with Acetaminophen in premedication.

\section{OBJECTIVES}

\section{General objective}

To determine the effect of Midazolam together with Acetaminophen in premedication.

\section{Specific objectives}

- To survey the adequacy of premedication.

- To measure the level of uneasiness in the children.

- To identify the level of sedation acquired at the season of breathed in enlistment.

\section{Methodology and Materials}

This was a descriptive observational study was conducted at the department of Anaesthesiology in 250 bedded general hospital, Pabna, Bangladesh during the period from the study during January 2017 to December, 2018. A total of 100 patients, below 8 years of age and either sex who were scheduled for elective surgery or diagnostic procedures requiring anaesthesia techniques and prescribed anxiolytic premedication with Midazolam were included in the study. The presence of a known malignancy or of a mental disease was considered as an exclusion criterion. All patients or their representatives provided written informed consent. During the preoperative assessment, the anaesthesiologist gathered demographic information aside from the usual information for the anaesthesia record. The patients were assessed by the attending anaesthetist, and given the modified Yale scale. Patients were taken on a stretcher to the operating room, and after setting up basic monitoring, inhaled induction was initiated using sevoflurane. At that point (1min), the Richmond-RASS sedation-agitation scale was applied. A characterization of the population, social and demographic data was performed for the statistical analysis, using descriptive statistical tools.

\section{RESUltS}

A total of 100 children included in this study. The mean BMI of the children were $15 \pm 3$. The age of the children was > 08 years. Of them, $30 \%$ were females and $70 \%$ were males. The application of the Yale scale showed adequate sedation and anxiolytics in $60 \%$ of the patients. It is worth noting that at the time of the evaluation, the time elapsed between the moment the drug was given until the scale was applied was within an ideal range $(15-90 \mathrm{~min})$ only in $25 \%$ of the children. Moreover, of the 55 patients in whom the administration interval was appropriate, $60 \%$ were found to be anxious, and $40 \%$ were not anxious. When the sedation-anxiolytics scale was applied at the time the children were separated from their parents, it showed that $61 \%$ of the children were indifferent or quiet, anious, not violent were $15 \%$, frequent movement were $08 \%$, agglessive, violent were $05 \%$, very agitated were $04 \%$, sleepy were $03 \%$, deep sedation was $01 \%$ At this stage, there was no evaluation of the time elapsed from the moment the Midazolam-acetaminophen mix was given. The Richmond-RASS scale applied at the time of induction showed that $65 \%$ of the patients had adequate sedation (score between -1 and 0 ), which resulted in easy and quiet acceptance of the facemask. 
Table-1: Demographic characteristics of the patients. $(n=100)$

\begin{tabular}{|l|l|l|}
\hline Variable & Description & $\%$ \\
\hline Age & $>8$ Years & \\
\hline BMI & $15 \pm 3$ & \\
\hline Male & 70 & 70 \\
\hline Female & 30 & 30 \\
\hline Prior Anesthesia-Yes & 35 & 35 \\
\hline Prior Anesthesia-No & 65 & 65 \\
\hline
\end{tabular}

Table-2: Comparison between Emotional Expressivity \& Interaction with relatives

\begin{tabular}{|l|l|}
\hline Emotional expressivity & Interaction with relatives \\
\hline Happy & Has fun, sits still \\
\hline Natural with no visible expression & Seeks contact with realities \\
\hline Sad worried & Looks at relatives quietly, does not seek contact \\
\hline Apparent state of arousal & Activity \\
\hline Aroused, looks around confidently & Curious plays in the room \\
\hline Quiet withdrawn & Does not explore, sits close to relatives \\
\hline Cries and does not want others near & Pushes with hands and feet and tries to get away \\
\hline
\end{tabular}

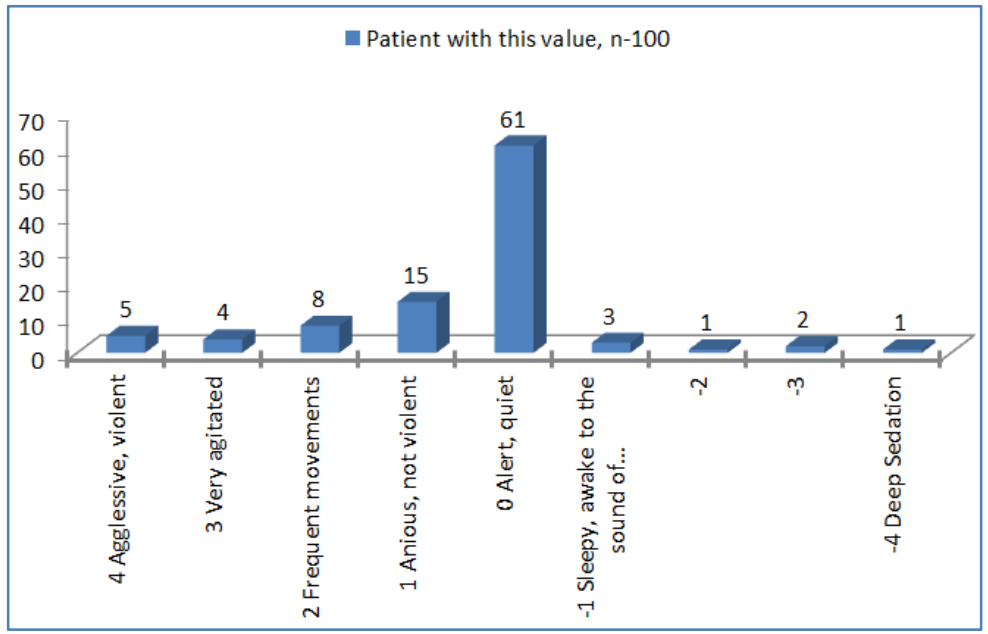

Fig-1: Data shows on sedation and anxiety scale

Table-3: Details about emotional expressivity.

\begin{tabular}{|l|l|}
\hline \multicolumn{2}{|l|}{ Emotional expressivity } \\
\hline Separation from parents: & Crying, Indifferent, Quiet \\
\hline Richmond-RASS scale: & $\begin{array}{l}\text { Aggressive, violent, Frequent movements Moderate } \\
\text { sedation. Opens eyes, does not fix gaze, Deep sedation, } \\
\text { does not respond to voice but does respond to physical } \\
\text { stimulus, Very deep sedation, does not respond to physical } \\
\text { stimulus }\end{array}$ \\
\hline
\end{tabular}

\section{DISCUSSION}

To achieve adequate sedation, one of the most effective pharmacological strategies is Midazolam which is one of the drugs of choice because of ease of administration, safety, good bioavailability when given orally, because it does not delay emergence from anaesthesia or the time to discharge from the recovery unit $[10,11]$. In the present study a total of 100 children were included to determine the effect of Midazolam together with Acetaminophen and the sedation scale were $61 \%$ of the children were indifferent or quiet, anious, not violent were $15 \%$, frequent movement were
$08 \%$, agglessive, violent were $05 \%$, very agitated were $04 \%$, sleepy were $03 \%$, deep sedation was $01 \%$. Reducing perioperative anxiety must be one of the most important objectives in pediatric anaesthesia, because anxiety has been associated with postoperative adverse events such as increased pain and negative behavioral changes, including psychomotor agitation at emergence, crying, disorientation, post-operative delirium, sleep disorders and avoidance behaviours in medical and hospital environments. The modified Yale scale was applied prior to separation from the parents, in the waiting room, in order to assess the presence or absence 
of anxiety at that point in time, and anxiety was found in $61 \%$ of patients. This might be explained because the time elapsed between the administration of oral Midazolam and that point in time was not adequate in most case. Another moment that might be traumatic and stressful for the child and might create psychological sequelae is aesthetic induction with the use of a facemask .In this study found that $65 \%$ of the patients showed good acceptance of the aesthetic induction, with good tolerance of the facemask; these results are similar to those reported by other authors. Intravenous Midazolam combined with other drugs such as fentanyl and protocol has been used for deep sedation in patients taken to magnetic resonance imaging. Oral Midazolam together with acetaminophen might probably be a good and safe option in less complex, non-painful diagnostic tests [12-15]. The similar result was found in the present study. In this study, the Richmond-RASS scale applied at the time of induction showed that $65 \%$ of the patients had adequate sedation (score between -1 and 0 ), which resulted in easy and quiet acceptance of the facemask.

\section{LIMITATIONS OF THE STUDY}

This study was conducted over a limited period of time. Only the entitled patient's got opportunity to participate in the study. So, the limited sample size, short duration, and a limited study area were the limitations of this study. So, with a large sample, vast area and large span of time, the study may be further conducted

\section{CONCLUSION AND \\ RECOMMENDATIONS}

From this study it is vivid that premedication with compounded Midazolam plus Acetaminophen used is useful. It reduces anxiety at the time when the children are separated from their parents, and it leads to good acceptance of inhaled anaesthesia induction, improving the whole experience, both for the children as well as for the parents.

\section{REFERENCES}

1. Haslet WHK and Dundee JW. Studies of drugs given before anaesthesia XIV: Two benzodiazepine derivatives chlor- diazepoxide and diazepam. Br I Anaesthesia 1968; 40:250-8.

2. Dundee JW, Hallidav NJ, Harper KW. MidazolamA review of its pharmacological properties and therapeutic use. Drugs 1984; 28:519-43.
3. Isik B, Baygin O, Kapci EG, Bodur H. The effects of temperament and behaviour problems on sedation failure in anxious children after midazolam premedication. Eur J Anaesthesiol. 2010;27:336- 40

4. Kaplan SA, Jack ML, Alexander K. Pharmacokinetic profile of diazepam in man following single intravenous and oral and chronic oral administrati6n. J. Pharmacol. Sci. 1973; 62:1789- 92.

5. Chasapakis G. Karatsaloar I, Partsaiidon A. Midazoloam (R O -21-3981) versus diazepam in premedication. Acta Anaes- thesia Helr. 1981; 15:68-72.

6. Mattila MAK, 5urinkovoinen S, Saila K. Midazolam and fat emulsion diazepam as intramuscular premedication - A double blind clinical trial. Acta Anaesth Scand. 1983; 27:345- 8.

7. Kiecolt-Glaser JK, Page GG, Marucha PT, MacCallum RC, Glaser R. Psychological influences on surgical recovery. Perspectives from psychoneuroimmunology. Am Psychol. 1998; 53:1209-18.

8. Pritchard MJ. Identifying and assessing anxiety in pre-operative patients. Nurs Stand. 2009; 23:35-40.

9. Bailey Jr PD, Bastien JL. Preinduction techniques for pediatric anesthesia. Curr Opin Anaesthesiol. 2005;18:265-9

10. Horgesheimer JJ, Pribble CG, Lugo RA. The effect of midazolam premedication on discharge time in pediatric patients undergoing general anesthesia for dental restorations. Pediatr Dent. 2001;23:491-4.

11. Fortier MA, Del Rosario AM, Martin SR, Kain ZN. Perioperative anxiety in children. PaediatrAnaesth. 2010;20:318-22.

12. Bozkurt P. Premedication of the pediatric patient anesthesia for the uncooperative child. Curr Opin Anaesthesiol. 2007; 20:211-5.

13. Vagnoli L, Caprilli S, Messeri A. Parental presence, clowns or sedative premedication to treat preoperative anxiety in children: what could be the most promising option? Paediatr Anaesth. 2010; 20:937-43.

14. Kazak Z, Sezer GB, Yilmaz AA, Ates Y. Premedication with oral midazolam with or without parental presence. Eur J Anaesthesiol. 2010; 27:347-52.

15. Maurice-Szamburski A, Auquier P, Viarre-Oreal V, Cuvillon P, Carles M, Ripart J. Effect of sedative premedication on patient experience after general anesthesia: a randomized clinical trial. JAMA. 2015; 313:916-25. 